\title{
Dual HER2 Blockade
}

\author{
Chair: \\ Christoph Thomssen ${ }^{a}$
}
Participants: Nagi S. El Saghir ${ }^{b}$ Prudence A. Francis $^{c}$ Jacek Jassem $^{d}$ Hope S. Rugo ${ }^{e}$ Michael Untch ${ }^{f}$

\author{
a Department of Gynecology, Martin-Luther-University, Halle/Saale, Germany; \\ ${ }^{b}$ Division of Hematology-Oncology, Department of Internal Medicine, American University of Beirut, Beirut, Lebanon; \\ ${ }^{c}$ Peter MacCallum Cancer Centre, Melbourne, Australia; \\ dDepartment of Oncology and Radiotherapy, Medical University of Gdańsk; Gdansk, Poland; \\ e Helen Diller Family Comprehensive Cancer Center, University of California San Francisco, San Francisco, CA, USA; \\ ${ }^{f}$ Gynecology Clinic, HELIOS Hospital Berlin Buch, Berlin, Germany
}

Although nearly doubling the response rates by the addition of a second anti-HER2 compound to the standard neoadjuvant treatment with trastuzumab (NeoALTTO [1], NeoSPHERE [2]), the interpretation of the adjuvant trials (ALTTO [3], APHINITY [4]) is more challenging. We asked experts from different countries around the world about their conclusions from that data. The discussion was performed under the assumption that the drug would be available in the respective countries.

\section{Question 1: Is the Neoadjuvant Treatment Approach Standard of Care for HER2-Positive Early Breast Cancer in Your Country or Your Department? What Is the Proportion of Neoadjuvant Treated Patients in HER2-Positive Disease?}

El Saghir: Neoadjuvant therapy for patients with HER2-positive early breast cancer has become a standard treatment approach and very common practice. We use it in all patients with locally advanced breast cancer, most patients with clinically positive axillary lymph nodes, and patients with a tumor size greater than $3 \mathrm{~cm}$. In patients with smaller tumors and negative axillary lymph nodes, it is only used if breast conserving therapy requires that we reduce the tumor size before surgery. We also employ it to make lymph node-positive patients amenable to sentinel lymph node biopsy and axilla conservation, in view of the high chance of achieving a pathologic complete response (pCR) [2]. This is practiced at our institution and in most university hospitals, but not necessarily in private practices throughout Lebanon.
Francis: The majority of patients with HER2-positive early breast cancer in Australia are still treated with an adjuvant approach outside of a clinical trial, with the neoadjuvant approach generally reserved for $15-20 \%$ of cases with larger tumor presentations. However, among surgeons in our center and nationally, there is increasing interest in a neoadjuvant approach.

Jassem: The neoadjuvant therapy is routinely used in patients with larger primary tumors or lymph node involvement at presentation.

Rugo: The majority of patients with HER $2+$ disease are treated with neoadjuvant chemotherapy combined with HER2-targeted therapy. Patients with very small tumors, particularly those who could be candidates for 12 weeks of weekly paclitaxel with 1 year of trastuzumab, will generally proceed with surgery before systemic therapy. We have treated patients who are elderly or have significant comorbidities with this regimen in the neoadjuvant setting to use this as a way to avoid more extensive therapy in excellent responders.

Untch: The neoadjuvant systemic treatment in patients with HER2-overexpressing primary tumors has been recommended by the National German Guideline for many years and has finally been also recommended by the St. Gallen Consensus Committee in the year 2017 [1]. In Germany, most of the patients in this situation are treated with neoadjuvant therapy. In our own department, all patients with HER2-positive disease receive neoadjuvant systemic treatment regardless of tumor size and clinical lymph node status.

\section{KARGER}

(๑) 2017 S. Karger GmbH, Freiburg

Fax +497614520714 


\section{Question 2: What Is Your Preferred Chemotherapy Regimen If You Are Planning Double HER2 Antibody Blockade?}

El Saghir: Our preferred regimen for NeoAdjuvant ChemoTherapy (NACT) is a dose-dense anthracycline-based taxane regimen. We use either AC-THP (THP = docetaxel plus trastuzumab plus pertuzumab; $\mathrm{AC}=$ doxorubicin $60 \mathrm{mg} / \mathrm{m}^{2}$ with cyclophosphamide $600 \mathrm{mg} / \mathrm{m}^{2}$ ) every 2 weeks, with G-CSF (granulocytecolony stimulating factor) support, followed by weekly paclitaxel $80 \mathrm{mg} / \mathrm{m}^{2}$ for 12 weeks, or docetaxel $100 \mathrm{mg} / \mathrm{m}^{2}$ every 3 weeks for 4 cycles. Pertuzumab plus trastuzumab are added concurrently with taxanes. We have the option of using epirubicin $90 \mathrm{mg} / \mathrm{m}^{2}$ instead of doxorubicin $60 \mathrm{mg} / \mathrm{m}^{2}$ (EC-THP). AC-THP is preferred over TCH/P (docetaxel, carboplatin, trastuzumab plus pertuzumab) because of a significant diarrhea side effect. For patients in whom we want to avoid anthracyclines, we use T/C (docetaxel + cyclophosphamide) or TC (docetaxel + carboplatin) with antiHER2.

Francis: The pCR rate reported with 6 cycles of TCH/P in the TRYPHAENA trial was excellent, particularly for hormone receptor-negative HER2-positive tumors. However, the risk of serious adverse events is significant, and so I believe that this regimen requires careful patient selection and growth factor support. The regimen utilized in the NeoSPHERE trial with an initial 4 cycles of THP seems a more broadly applicable regimen. In the NeoSPHERE trial, anthracycline therapy was given postoperatively prior to completing HER2-targeted therapy, but we might deliver anthracycline therapy preoperatively after 4 cycles of THP. Weekly paclitaxel plus trastuzumab plus pertuzumab is also utilized, in sequence with AC.

Jassem: Pertuzumab is not reimbursed in the neoadjuvant setting, and therefore not used. The most commonly used regimen in HER2-positive patients is 4 cycles of AC q 3 weeks followed by 12 cycles of weekly paclitaxel, concurrently with trastuzumab.

Rugo: We use both the sequential paclitaxel with double HER2 antibody blockade followed by doxorubicin and cyclophosphamide (THP/AC) as well as the docetaxel/carboplatin plus double antibody blockade (TCH/P) regimens. For younger patients with more extensive disease, we prefer the anthracycline-based regimen, and for older patients and those at higher risk for cardiac toxicity, we use TCH/P. One consideration for $\mathrm{TCH} / \mathrm{P}$ is the risk of serious diarrhea, which requires careful patient education and follow-up.

Untch: The preferred chemotherapy regimen in conjunction with double anti-HER2-blockade is 4 cycles of epirubicin, cyclophosphamide $\mathrm{q} 3 \mathrm{w} 90 / 600 \mathrm{mg} / \mathrm{m}^{2}$ followed by 12 -weekly paclitaxel $80 \mathrm{mg} / \mathrm{m}^{2}$ in addition to dual anti-HER2 blockade.

\section{Question 3: Would You Consider Double HER2 Antibody Blockade Using Trastuzumab and Pertuzumab plus Chemotherapy as Standard of Care in the Neoadjuvant Therapy of HER2-Positive Early Breast Cancer? Which Patients Are You Excluding from Such an Approach?}

El Saghir: Double HER2-antibody therapy using pertuzumab and trastuzumab plus chemotherapy is considered a standard therapy option in the neoadjuvant therapy of HER2-positive early breast cancer [2], and we practice it. Excluded are those patients who have small tumors, clinically negative lymph nodes, and hormone receptor-positive disease.

Francis: Pertuzumab is approved but not government-funded for neoadjuvant therapy in HER2-positive early breast cancer in Australia. Therefore, dual HER2-antibody blockade with chemotherapy is not a standard of care for all patients undergoing neoadjuvant therapy. Dual blockade would not generally be recommended for $\mathrm{cT} 1$ tumors or for lymph node-negative tumors unless the primary was large.

Jassem: Not until a survival benefit is proven.

Rugo: With current data, I would consider double HER2 antibody blockade plus chemotherapy to be the standard of care as neoadjuvant therapy for HER2-positive early-stage breast cancer. However, based on the current data from the APHINITY trial, patients with smaller node-negative and hormone receptor-positive tumors could be treated with chemotherapy plus trastuzumab with similar outcomes.

Untch: The dual anti-HER2 blockade with trastuzumab and pertuzumab + chemotherapy is the standard of neoadjuvant therapy for patients with HER2-positive early breast cancer. Patients who have unsuspicious axillary lymph nodes by palpation and sonography might not be candidates for this approach. Nevertheless, it should be taken into account that in about $30 \%$ of patients with unsuspicious axillary lymph nodes histology shows axillary node involvement. Because of this reason, patients with unsuspicious lymph nodes in the axilla should also be offered the possibility of dual anti-HER2 blockade, especially with other risk criteria: hormone negativity, younger age, etc.

\section{Question 4: Would You Prolong the Double Blockade after Ending Neoadjuvant Therapy instead of Giving Trastuzumab Only (to Complete 1 Year of Therapy)?}

El Saghir: We recommend continuing pertuzumab, in addition to trastuzumab, beyond the neoadjuvant phase of therapy in patients who had a primary locally advanced breast cancer, or have positive axillary lymph nodes at diagnosis documented by fine needle aspiration or core biopsy or show treatment effects at pathology 
of removed lymph nodes, or hormone receptor-negative tumors. The APHINITY trial has shown no significant benefit in the adjuvant setting for patients with smaller tumors, negative lymph nodes, and hormone receptor-positive tumors [4]. The APT trial has shown that patients with tumors that are $<3 \mathrm{~cm}$, lymph node-negative, and hormone receptor-positive have excellent survival results with only paclitaxel and trastuzumab [5]; theoretically, a little more can be obtained by adding pertuzumab in such patient groups.

Francis: Generally, I would cease the double blockade after completion of the neoadjuvant therapy component and would be more likely to administer trastuzumab only for the postoperative component, combined with endocrine therapy if hormone receptor-positive.

Jassem: Our practice is continuing trastuzumab alone for a total of 1 year.

Rugo: It is hard to know how much benefit is obtained from continuing pertuzumab for 1 year, as optimal duration has not been studied. Right now we do not use 1 year of pertuzumab, instead confining use to either the neoadjuvant setting or the adjuvant setting in patients who would otherwise have received neoadjuvant therapy if given the option. A patient who achieves a pCR with neoadjuvant therapy including double antibody therapy could perhaps safely discontinue pertuzumab at the time of surgery. For those not achieving a pCR, either continuing pertuzumab or perhaps changing HER2-targeted therapy may be the best option. We will have to wait for the outcome of the post-neoadjuvant trial studying trastuzumab-emtansine to understand the impact of the antibody drug conjugate in this setting, and extended adjuvant therapy with neratinib in patients with hormone receptor-positive, HER2-positive disease is also an option [6].

Untch: In patients who have pCR after neoadjuvant therapy with dual anti-HER2 blockade, there is no need for giving dual antiHER2 blockade after surgery. The standard of care is completion of anti-HER2 treatment with another 6 months of trastuzumab.

\section{Question 5: Should the Adjuvant Treatment Always Contain Both Antibodies? To Which Patients Would You Restrict Double HER2 Antibody Blockade?}

El Saghir: Not all patients should be offered dual anti-HER2 antibody therapy. The benefit regarding invasive disease-free survival (IDFS) for addition of pertuzumab to trastuzumab in the APHINITY trial was limited (3-year overall IDFS is $94.1 \%$ vs. $93.2 \%, \mathrm{p}=$ 0.045 , absolute benefit $0.9 \%$ ); patients with node-positive disease had an IDFS of $92 \%$ versus $90.2 \%$ ( $p=0.02$, absolute benefit $1.8 \%$ ). Patients with node-negative disease had an IDFS of $98.4 \%$ versus $97.5 \%$, with an absolute benefit of only $0.9 \%$. Hormone receptornegative patients had a modest benefit with an IDFS of $92.8 \%$ versus $91.2 \%$; thus, the absolute benefit was $1.6 \%$ [4]. Therefore, use of dual anti-HER2 adjuvant therapy should be restricted to patients with large tumors, multiple positive nodes, and negative hormone receptors. Longer follow-up of the APHINITY trial will give us more answers as to which patients have a significant benefit from dual pertuzumab + trastuzumab in the adjuvant setting.

Francis: I do not think that adjuvant treatment should always contain both antibodies. There were many patients with tumors at relatively low risk for relapse enrolled in the APHINITY trial, which makes the overall results difficult to interpret currently for a patient with a high-risk tumor. It is plausible that meaningful absolute improvement in outcomes could be obtained in patients with multiple positive lymph nodes, for example. Future (neo-)adjuvant breast cancer trials involving escalation of therapy should avoid recruiting patients at low risk for recurrence. Neoadjuvant approaches offer better opportunities for tailoring treatment.

Jassem: Not until a survival benefit is proven.

Rugo: We just do not have enough information yet. Based on the current APHINITY analysis, it appears that the patients who benefit the most are those with higher risk and more HER2-enriched disease, marked by node positivity and endocrine negativity. I would not use double antibody therapy in patients who could be treated with the so-called APT regimen, including weekly paclitaxel and 1 year of trastuzumab. The APT trial included patients with node-negative disease up to $3 \mathrm{~cm}$. Based on the data we have today, I would restrict double HER2 antibody blockade to patients with higher risk disease.

Untch: Increasingly, the data from the APHINITY study offer the rationale for giving dual anti-HER2 blockade also in the adjuvant setting. Especially for patients with histologically proven involvement of axillary lymph nodes, this approach should be the standard.

\section{Question 6: Do You See Any Indication for the Use of Antibodies Alone (without Chemotherapy) in Any Patient?}

El Saghir: Neoadjuvant therapy with dual antibodies and chemotherapy yields more pCR in the neoadjuvant setting. We have no data to support dual antibodies alone in the adjuvant setting. However, we recommend dual anti-HER2 therapy in combination with hormonal therapy in the presence of contraindications for the use of chemotherapy. We need better biomarkers and studies to define the subset of patients that can be spared chemotherapy.

Francis: I think that the possibility to treat selected HER2-positive breast cancers with antibodies alone is an important future option. This appears most likely to succeed in the hormone receptornegative and/or HER2-enriched tumor subtype. This approach is not yet being implemented in routine practice, but I believe that 
de-escalation strategies in HER2-positive breast cancer are an important area for study, and hopefully specific clinical trials will be developed.

Jassem: This should not be a standard in any setting until noninferiority with standard therapy is proven. However, this therapy may be considered in patients with apparent contraindications to chemotherapy.

Rugo: I would not use antibody therapy alone as treatment for early-stage breast cancer. In a patient with a smaller hormone receptor-positive tumor who is not a candidate for chemotherapy, I would consider trastuzumab with endocrine therapy.

Untch: Some subgroup analyses from large trials have indicated that with antibody treatment alone we can reach pCRs in a substantial percentage of patients. Unfortunately, up until now there are no biomarkers to prove in which cases chemotherapy can be omitted. Therefore, in patients with HER2-positive disease, chemotherapy + anti-HER2 chemotherapy is the standard of care.

\section{Question 7: What Are the Future Perspectives of Double HER2 Antibody Blockade in Early Breast Cancer?}

El Saghir: Although expectations were high, and a press release indicated that the APHINITY trial has met its primary endpoint, the presented and published results of escalation of adjuvant therapy with dual HER2 antibody blockade in the APHINITY trial were disappointing. Even subgroups of patients with positive lymph nodes or hormone receptor-negative tumors had clinical benefits on a relatively small scale. We need to focus research on patients with 'relatively advanced early' stage disease and less hormone-dependent disease where we should escalate therapy. Genomics studies on HER2-positive patients may turn out to be helpful. We need to have biomarkers that allow us to de-escalate therapy while preserving benefit and reducing side effects $[7,8]$.

Francis: Given the excellent results with chemotherapy plus trastuzumab in the majority of patients with HER2-positive early breast cancer, we need better methods to identify very early in the course of neoadjuvant therapy those unlikely to experience optimal outcomes with this therapy, who would then be candidates for escalation to dual HER2-targeted therapy, instead of increasing the number of HER2 therapies for all patients. In the future, we may also need to consider different trials/approaches for hormone receptorpositive and hormone receptor-negative HER2-positive tumors.

Jassem: It is very unlikely that double blockade with currently available agents will ever become a new standard for the majority of HER2-positive breast cancer patients. This may change though should new biomarkers better select patients for individualized approaches.

Rugo: We need longer follow-up from the APHINITY trial to understand which subpopulation benefits from dual antibody blockade, and to fully evaluate the impact of dual versus single antibody blockade on overall survival. It is critical that we carefully evaluate these factors to effectively apply therapy without overtreating the entire HER2 population of patients, many of whom may not require dual antibody blockade. To date, we have not been successful in identifying biomarkers of greater response to pertuzumab, but clinical-pathologic factors may allow some degree of stratification. We also need to understand the optimal duration of dual antibody therapy, although this was not evaluated in the APHINITY trial. Lastly, we need to understand the additional impact of extended HER2-targeted therapy with neratinib in patients who have received pertuzumab [6].

Untch: Patients with pCR after double anti-HER2 blockade might be candidates for no further anti-HER2 treatment. This has to be proven in randomized trials.

Patients with residual disease after anti-HER2 blockade were randomized in the KATHERINE study to be treated with TDM1. The first results will probably be presented at ASCO in 2018.

\section{Participants}

Prof. Dr. Nagi S. El Saghir

Division of Hematology-Oncology, Department of Internal Medicine

American University of Beirut

P.O. Box 11-0236, Riad-El-Solh, Beirut 1107 2020, Lebanon

nagi.saghir@aub.edu.lb

Prof. Dr. Prudence A. Francis

Peter MacCallum Cancer Centre

305 Grattan Street, Melbourne VIC 3000, Australia

prue.francis@petermac.org

Prof. Dr. Jacek Jassem

Department of Oncology and Radiotherapy

Medical University of Gdańsk

ul. Dębinki 7, 80-211Gdansk, Poland

jjassem@gumed.edu.pl

Hope S. Rugo, M.D.

Helen Diller Family Comprehensive Cancer Center

University of California San Francisco

1600 Divisadero St, San Francisco, CA, 94115, USA

hope.rugo@ucsf.edu

Prof. Dr. Michael Untch

Gynecology Clinic, HELIOS Hospital Berlin Buch

Schwanebecker Chaussee 50, 13125 Berlin, Germany

michael.untch@helios-kliniken.de 


\section{References}

1 Baselga J, Bradbury I, Eidtmann H, et al.; NeoALTTO Study Team: Lapatinib with trastuzumab for HER2positive early breast cancer (NeoALTTO): a randomised, open-label, multicentre, phase 3 trial. Lancet 2012;379:633-640.

2 Gianni L, Pienkowski T, Im YH, et al: 5-year analysis of neoadjuvant pertuzumab and trastuzumab in patients with locally advanced, inflammatory, or earlystage HER2-positive breast cancer (NeoSphere): a multicentre, open-label, phase 2 randomised trial. Lancet Oncol 2016;17:791-800.

3 Piccart-Gebhart M, Holmes E, Baselga J, et al: Adjuvant lapatinib and trastuzumab for early human epidermal growth factor receptor 2-positive breast cancer: results from the randomized phase III adjuvant lapatinib and/or trastuzumab treatment optimization trial. J Clin Oncol 2016;34:1034-1042.
4 von Minckwitz G, Procter M, de Azambuja E, et al.; APHINITY Steering Committee and Investigators: Adjuvant pertuzumab and trastuzumab in early HER2positive breast cancer. N Engl J Med 2017;377:122-131.

5 Tolaney SM, Barry WT, Dang CT, et al: Adjuvant paclitaxel and trastuzumab for node-negative, HER2-positive breast cancer. N Engl J Med 2015;372:134-141.

6 Chan A, Delaloge S, Holmes FA, et al.; ExteNET Study Group: Neratinib after trastuzumab-based adjuvant therapy in patients with HER2-positive breast cancer (ExteNET): a multicentre, randomised, double-blind, placebo-controlled, phase 3 trial. Lancet Oncol 2016; 17:367-377.
Cardoso F, Harbeck N, Barrios CH, et al: Research needs in breast cancer. Ann Oncol 2017;28:208-217.

8 Curigliano G, Burstein HJ, P Winer E, et al.; St. Gallen International Expert Consensus on the Primary Therapy of Early Breast Cancer 2017: De-escalating and escalating treatments for early-stage breast cancer: the St. Gallen International Expert Consensus Conference on the Primary Therapy of Early Breast Cancer 2017. Ann Oncol 2017;28:1700-1712. 